\title{
Recurrent skin and soft tissue infections in HIV-infected patients during a 5-year period: incidence and risk factors in a retrospective cohort study
}

\author{
Vagish Hemmige ${ }^{1 *}$, Moira McNulty², Ethan Silverman ${ }^{2}$ and Michael Z. David ${ }^{2,3}$
}

\begin{abstract}
Background: Skin and soft tissue infections (SSTIS) are common in the era of community-associated methicillin resistant Staphylococcus aureus among HIV-infected patients. Recurrent infections are frequent. Risk factors for recurrence after an initial SSTI have not been well-studied.

Methods: Retrospective cohort study, single center, 2005-2009. Paper and electronic medical records were reviewed by one of several physicians. Subjects with initial SSTI were followed until the time of SSTI recurrence. Standard descriptive statistics were calculated to describe the characteristics of subjects who did and did not develop a recurrent SSTI. Kaplan-Meier methods were used to estimate the risk of recurrent SSTI. A Cox regression model was developed to identify predictors of SSTI recurrence.
\end{abstract}

Results: 133 SSTIS occurred in 87 individuals. 85 subjects were followed after their initial SSTI, of whom 30 (35.3\%) had a recurrent SSTI in 118.3 person-years of follow-up, for an incidence of second SSTI of 253.6 SSTIs/1000 person-years (95\% Cl 166.8-385.7). The 1-year Kaplan-Meier estimated risk of a second SSTI was $29.2 \%$ (95 \% Cl 20.3-41.0 \%), while the 3-year risk was $47.0 \%$ (95\% Cl 34.4-61.6\%). Risk factors for recurrent SSTI in a multivariable Cox regression model were non-hepatitis liver disease (HR 3.44; $95 \% \mathrm{Cl} 1.02-11.5 ; p=0.05)$, the presence of an intravenous catheter (HR 6.50; $95 \% \mathrm{Cl} 1.47-28.7 ; p=0.01$ ), and a history of intravenous drug use (IVDU) (HR 2.80; $95 \%$ Cl 1.02-7.65; $p=0.05$ ); African-American race was associated with decreased risk of recurrent SSTI (HR 0.12; $95 \% \mathrm{Cl} 0.04-0.41 ; p<0.01)$. Some evidence was present for HIV viral load $\geq 1000$ copies $/ \mathrm{mL}$ as an independent risk factor for recurrent SSTI (HR 2.21; $95 \% \mathrm{Cl} 0.99-4.94 ; p=0.05)$. Hemodialysis, currently taking HAART, CD4+ count, trimethoprim-sulfamethoxazole or azithromycin use, initial SSTI type, diabetes mellitus, incision and drainage of the original SSTI, or self-report of being a man who has sex with men were not associated with recurrence.

Conclusion: Of HIV-infected patients with an SSTI, nearly $1 / 3$ had a recurrent SSTI within 1 year. Risk factors for recurrent SSTI were non-hepatitis liver disease, intravenous catheter presence, a history of IVDU, and non-African-American race. Low CD4+ count was not a significant risk factor for recurrence.

Keywords: HIV, Epidemiology, Skin and soft tissue infections, MRSA

\footnotetext{
* Correspondence: vagish.hemmige@bcm.edu

'Division of Infectious Diseases, Department of Medicine, Baylor College of Medicine, 6620 Main, Suite 1375, Houston, TX 77030, USA

Full list of author information is available at the end of the article
}

(c) 2015 Hemmige et al. Open Access This article is distributed under the terms of the Creative Commons Attribution 4.0 International License (http://creativecommons.org/licenses/by/4.0/, which permits unrestricted use, distribution, and reproduction in any medium, provided you give appropriate credit to the original author(s) and the source, provide a link to the Creative Commons license, and indicate if changes were made. The Creative Commons Public Domain Dedication waiver (http://creativecommons.org/publicdomain/zero/1.0/) applies to the data made available in this article, unless otherwise stated. 


\section{Background}

In 1998, the first increase in incidence of methicillin resistant Staphylococcus aureus (MRSA) infections in people without previous contact with the health care system was reported in children in Chicago [1]. Community-associated (CA-MRSA) infections were subsequently identified in other groups in the United States, especially marginalized populations, and CA-MRSA quickly became the leading cause of skin and soft tissue infections (SSTIs) with community onset among incarcerated populations and among impoverished urban populations [2, 3]. The advent of the CA-MRSA era dramatically affected the epidemiology of SSTIs, with a doubling in the number of SSTIs seen at safety net hospital emergency rooms [2].

Patients with human immunodeficiency virus (HIV) infection are particularly prone to infection with CAMRSA because they are immunocompromised and because of demographic, behavioral, and socioeconomic factors as well as frequent exposures to the health care system $[4,5]$. We have recently evaluated risk factors for a first SSTI among $511 \mathrm{HIV}$-infected patients in Chicago [6]. In that study, accounting for follow-up time, chronic skin conditions such as psoriasis and lymphedema, presence of an intravenous catheter, and lack of virologic suppression were most strongly associated with initial SSTI occurrence. There was also a trend toward an increased risk of SSTI in subjects receiving health insurance through Medicaid, the governmental health insurance program for the poor and disabled, and subjects with sexually transmitted diseases (STDs). Other studies have identified different risk factors [4], though our study differed methodologically from many other studies in that we identified all SSTIs which were documented in the medical record, not just SSTIs which were culture positive for $S$. aureus. This is important because in our study we found that cultures were more likely to be sent during an SSTI episode in patients with advanced immunocompromise [6]. Therefore, relying on culture data as a criterion for detection of an SSTI in retrospective studies may lead to biased conclusions about the epidemiology of SSTIs in the HIV-infected population. Although a number of anecdotal reports have suggested that recurrent SSTIs are common among HIV-infected patients [7, 8], few studies have examined the incidence of and risk factors for recurrent SSTIs in the HIV-infected population in the current era of CA-MRSA [9-12]. Accordingly, we set out to examine the risk factors for SSTI recurrence in an HIVinfected population receiving care at an urban tertiary care center clinic in the CA-MRSA era.

\section{Methods}

\section{Study design}

We performed a retrospective study of $511 \mathrm{HIV}$-infected adults ( $>18$ years of age) who received their primary
HIV care at the University of Chicago Medical Center's (UCMC) Infectious Diseases Clinic for any period of time between January 1, 2005 and December 31, 2009. A description of this cohort has previously been published elsewhere [6]. SSTI was defined based on a physician diagnosis identified in medical record review. Recurrent SSTI was defined as either an SSTI at a new site or recurrence at the original site greater than 30 days after the initial SSTI with resolution of the signs and symptoms of the original infection in the interim. Individuals eligible for this study were those in the cohort who developed an SSTI and who accrued further follow-up time in the clinic after the initial SSTI. Subjects contributed follow-up time to this study from the day after the initial SSTI until either the occurrence of a first SSTI recurrence or until censoring. The study was approved by the Institutional Review Board of the Biological Sciences Division of the University of Chicago. Requirement for informed consent was waived.

\section{Statistical methods}

Proportions between groups were compared by the $\chi^{2}$ or Fisher's exact test, as appropriate (i.e. if any cell in the appropriate $2 \times \mathrm{N}$ categorical table contained five or fewer subjects). Differences between groups of continuous outcomes were compared using the $t$-test or the Wilcoxon rank-sum test. Cox regression was used for time-to event data to calculate the hazard ratio (HR) with $95 \%$ confidence interval (CI) associated with various patient characteristics [13]. The overall fit of potential Cox models was assessed via a plot of the Nelson-Aalen cumulative hazard against Cox-Snell residuals $[14,15]$. The proportional hazards assumption was verified in potential models via the use of log-log plots, and the presence or lack of influential observations was verified by calculating DFBETAs for each potential predictor [15]. Incidence rates were calculated using cluster-adjusted Poisson regression [16]. Based on exploratory analysis, we chose to categorize viral load as a binary variable with cutoff of 1000 copies/mL. CD4+ count was similarly categorized as a binary variable with cutoff of 100 cells $/ \mathrm{mL}$. Patients who had greater than 365 days without a measured CD4+ count or HIV viral load (VL) were deemed to have been lost to follow-up for that period and were interval censored in the statistical analysis [17].

All analyses were performed using Stata 12 (Statacorp; College Station, TX).

\section{Results}

\section{Population characteristics}

Of the 511 subjects in the initial cohort, 87 individuals (17.0 \%) experienced at least one SSTI, as described elsewhere [6]. Two subjects from the cohort had no followup time after the initial SSTI, so 85 subjects were 
included in the analysis reported in this study. The demographics of this cohort are shown in Table 1. The 85 subjects contributed 118.3 person-years of follow-up, with subjects censored at loss to follow-up or at the time of recurrent SSTI.

Of the initial SSTIs, 50 (58.8\%) were abscesses, 27 $(31.8 \%)$ were furuncles, and $8(9.4 \%)$ were cellulitides. Incision and drainage of the initial SSTI was performed in 28 cases (32.9\%). Only 23/85 initial SSTIs were associated with culture data, one of which was culture-negative. $S$. aureus was an identified pathogen in 15 initial SSTIs, and MRSA in 11 of these. Pseudomonas aeruginosa was the only other pathogen identified in more than one patient, having been identified in 3 SSTIs. All cultures were from an infected skin site.

Of the 85 initial SSTIs, 20 (23.5 \%) were treated with combination antimicrobial drug therapy. The most common combination used was ciprofloxacin and clindamycin, used in 3 patients ( $3.5 \%$ of all patients, $15 \%$ of patients receiving combination therapy). Of the 65 who did not receive combination antimicrobial drug therapy, 20 (23.5\% of all initial SSTIs) received a beta-lactam, 21 $(24.7 \%)$ received clindamycin, 8 (9.4\%) received trimethoprim-sulfamethoxazole, $5(5.9 \%)$ received a fluoroquinolone, $2(2.4 \%)$ received vancomycin, and 3 (3.5\%) received no antimicrobial drug therapy. Whether or not antimicrobial drug therapy was prescribed for the initial SSTI was not documented in the medical records of six $(7.1 \%)$ patients. As documented in the medical record, no subject underwent attempts at decolonization after the initial SSTI.

A total of 30 subjects among the 85 who had follow-up (35.3 \%) experienced at least one recurrent SSTI during follow-up. Using Kaplan-Meier methods, the estimated proportion of individuals who would have experienced a recurrent SSTI if all individuals had been followed for one year was $29.2 \%$ (95\% CI $20.3 \%-41.0 \%$ ), while the 3-year risk was $47.0 \%$ (95\% CI 34.4 \%-61.6 \%). Figure 1 shows the estimated Kaplan-Meier cumulative incidence of SSTIs over time. The incidence of second SSTI was 253.6 SSTIs/1000 person-years (95 \% CI 166.8-385.7). Fourteen subjects experienced multiple recurrences $(16.5 \%$ of all subjects with initial SSTI; $46.7 \%$ of subjects with at least one recurrence).

A number of variables assessed as potential risk factors for SSTIs were time-varying. These variables were therefore not assumed to be present throughout the followup period; instead, they were calculated as present or absent on each day of follow-up. For example, $79.8 \%$ of follow-up time accrued to individuals on Medicaid, $1.6 \%$ to individuals with a catheter, $24.7 \%$ to individuals taking trimethoprim-sulfamethoxazole prophylaxis, and $12.0 \%$ to individuals taking azithromycin prophylaxis. Highly active antiretroviral therapy (HAART) was being given $77.1 \%$ of the time, and the cohort had an HIV VL $<1000$ copies $/ \mathrm{mL}$ on $60.0 \%$ of days. The diagnosis of certain comorbid conditions was assessed similarly, with $14.6 \%$ of follow-up time occurring in subjects with diabetes, and $7.8 \%$ with cancer (Table 2).

\section{Characteristics of recurrent SSTIs}

The median recurrent SSTI occurred at 144 days after the initial SSTI (interquartile range [IQR], 89-377). Trimethoprim-sulfamethoxazole prophylaxis was being taken by $5 / 30$ subjects ( $16.7 \%$ ) at the time of SSTI, and 3/6 (50\%) individuals with CD4+ count $<200$ cells $/ \mathrm{mm}^{3}$ at the time of SSTI were taking trimethoprim-sulfamethoxazole. The median CD4+ count at the time of recurrent SSTI was 403.5 (IQR 239-529), and the median VL was 1475 copies/ $\mathrm{mL}$ (IQR undetectable-27800). We found that 26/30 (86.7 \%) SSTI recurrences occurred in patients with CD4+ count $>100$ cells $/ \mathrm{mm}^{3}$. Of the recurrent infections, 17 were abscesses (56.7\%), 9 were cellulitis (30.0\%), and 3 were furuncles (10.0\%), and the type of SSTI was undocumented in one case. 13 of 30 (43.3\%) recurrent infections met Centers for Disease Control and Prevention (CDC) criteria for a community-associated SSTI (CA-SSTI) [18]. The primary reason that an SSTI was not classified as a CASSTI was hospitalization within the prior year, which was the case in 12/30 (40.0 \%) patients.

Culture data were only obtained for 8 of the recurrent SSTIs, and of those, 6 of the 8 (75\%) were confirmed $S$. aureus infections, with 4 of the $6(67 \%)$ being MRSA. Of those 4 infections, 3 were clindamycin-susceptible MRSA, and all four were susceptible to trimethoprimsulfamethoxazole. Of the 6 culture-proven $S$. aureus infections, only 1 MRSA SSTI met CDC criteria for being a CA-SSTI. The other two SSTIs were caused by Proteus and a coagulase negative staphylococcus species. Three of the six recurrent SSTIs caused by S. aureus were associated with culture data for the initial SSTI; all three initial SSTIs were caused by S. aureus as well. In two patients, both infections were causes by MRSA, and in one patient, both infections were causes by MSSA.

Therapy in cases of recurrent SSTI was with a betalactam in five cases $(16.7 \%)$, combination therapy in seven cases $(23.3 \%)$, clindamycin in four cases (13.3\%), TMP-SMX in three cases $(10 \%)$, levofloxacin in one case $(3.3 \%)$, and vancomycin in one case $(3.3 \%)$. Five patients $(16.7 \%)$ received no systemic antibacterial therapy, and antibiotic therapy was not documented in four cases (13.3\%).

\section{Predictors of Recurrent SSTI}

The results of univariate Cox regression with possible predictors of SSTI are listed in Table 3. At any given time point, the presence of an intravascular catheter (HR 4.62; $95 \%$ confidence intervals $[\mathrm{CI}] 1.08-19.7 ; p=0.04$ ), chronic 
Table 1 Baseline characteristics of individuals who did and did not develop a recurrent SSTI during the study period

\begin{tabular}{|c|c|c|c|}
\hline Baseline variable & No recurrent SSTI $(n=55)$ & Had recurrent SSTI $(n=30)$ & Total $(n=85)$ \\
\hline \multicolumn{4}{|l|}{ Sex } \\
\hline Male & $30(65.2 \%)$ & $16(34.8 \%)$ & $46(54.1 \%)$ \\
\hline Female & $25(64.1 \%)$ & $14(35.9 \%)$ & $39(45.9 \%)$ \\
\hline \multicolumn{4}{|l|}{ Sexual behavior } \\
\hline Male & $30(65.2 \%)$ & $16(34.8 \%)$ & 46 \\
\hline Sex with men and women & $6(75.0 \%)$ & $2(25.0 \%)$ & $8(17.4 \%)$ \\
\hline Sex with men & $11(64.7 \%)$ & $6(35.3 \%)$ & $17(36.7 \%)$ \\
\hline Sex with women & $13(61.9 \%)$ & $8(38.1 \%)$ & $21(45.7 \%)$ \\
\hline Female & $25(64.1 \%)$ & $14(35.9 \%)$ & 39 \\
\hline Sex with women & $1(100 \%)$ & $0(0 \%)$ & $1(2.6 \%)$ \\
\hline Sex with men & $24(63.2 \%)$ & $14(36.8 \%)$ & $38(97.4 \%)$ \\
\hline Mean initial age (sd) & $40.7(1.5)$ & $40.1(1.9)$ & $40.5(1.2)$ \\
\hline Median initial CD4 (IQR) & $363(180-553)$ & $351(242-542)$ & $353(199-552)$ \\
\hline \multicolumn{4}{|l|}{ Initial VL at time of first SSTI } \\
\hline$<1000$ & $28(70.0 \%)$ & $12(30.0 \%)$ & $40(47.1 \%)$ \\
\hline $1000+$ & $27(60.0 \%)$ & $18(40.0 \%)$ & $45(52.9 \%)$ \\
\hline \multicolumn{4}{|l|}{ IVDU history } \\
\hline No & $49(67.1 \%)$ & $24(32.9 \%)$ & $73(86.9 \%)$ \\
\hline Yes & $5(45.4 \%)$ & $6(54.6 \%)$ & $11(13.1 \%)$ \\
\hline \multicolumn{4}{|l|}{ Inhaled drug use } \\
\hline No & $41(64.1 \%)$ & $23(35.9 \%)$ & $64(76.2 \%)$ \\
\hline Yes & $13(65.0 \%)$ & 7 (35.0 \%) & $20(23.8 \%)$ \\
\hline \multicolumn{4}{|l|}{ Methamphetamine use } \\
\hline No & $54(65.1 \%)$ & $29(35.9 \%)$ & $83(98.8 \%)$ \\
\hline Yes & $0(0 \%)$ & $1(100 \%)$ & $1(1.2 \%)$ \\
\hline \multicolumn{4}{|l|}{ Incarceration } \\
\hline No & $47(61.8 \%)$ & $29(38.2 \%)$ & $76(90.5 \%)$ \\
\hline Yes & $7(87.5 \%)$ & $1(12.5 \%)$ & $8(9.5 \%)$ \\
\hline \multicolumn{4}{|l|}{ Race } \\
\hline African-American & $52(67.5 \%)$ & $25(32.5 \%)$ & 77 (91.7%) \\
\hline Caucasian & $2(40.0 \%)$ & $3(60.0 \%)$ & $5(5.9 \%)$ \\
\hline Hispanic & $1(50.0 \%)$ & $1(50.0 \%)$ & $2(2.4 \%)$ \\
\hline \multicolumn{4}{|l|}{ Educational status } \\
\hline Did not complete high school & $5(83.3 \%)$ & $1(16.7 \%)$ & $6(22.2 \%)$ \\
\hline High school graduate & $1(33.3 \%)$ & $2(66.7 \%)$ & $3(11.1 \%)$ \\
\hline Some college & $8(66.7 \%)$ & $4(33.3 \%)$ & $12(44.4 \%)$ \\
\hline College graduate & $4(66.7 \%)$ & $2(33.3 \%)$ & $6(22.2 \%)$ \\
\hline \multicolumn{4}{|l|}{ Employment during follow-up } \\
\hline No & $32(64.0 \%)$ & $18(36.0 \%)$ & $50(59.5 \%)$ \\
\hline Yes & $22(64.7 \%)$ & $12(35.3 \%)$ & $34(40.5 \%)$ \\
\hline \multicolumn{4}{|l|}{ Chronic skin disease } \\
\hline No & $38(69.1 \%$ & $17(30.9 \%)$ & 55 (64.7\%) \\
\hline Yes & 17 (56.7 \%) & 13 (43.3\%) & 30 (35.3 \%) \\
\hline
\end{tabular}


Table 1 Baseline characteristics of individuals who did and did not develop a recurrent SSTI during the study period (Continued)

\begin{tabular}{|c|c|c|c|}
\hline \multicolumn{4}{|l|}{ Hepatitis C } \\
\hline No & 47 (64.4\%) & $26(35.6 \%)$ & $73(85.9 \%)$ \\
\hline Yes & $8(66.7 \%)$ & $4(33.3 \%)$ & $12(14.1 \%)$ \\
\hline \multicolumn{4}{|l|}{ Non-hepatitis liver disease } \\
\hline No & $54(67.5 \%)$ & $26(32.5 \%)$ & $80(94.1 \%)$ \\
\hline Yes & $1(20.0 \%)$ & $4(80.0 \%)$ & $5(5.9 \%)$ \\
\hline \multicolumn{4}{|l|}{ Initial SSTI type } \\
\hline Abscess & $28(56.0 \%$ & $22(44.0 \%)$ & $50(58.8 \%)$ \\
\hline Furuncle & $20(74.1 \%)$ & $7(25.9 \%)$ & $27(31.8 \%)$ \\
\hline Cellulitis & $7(87.5 \%)$ & $1(12.5 \%)$ & $8(9.4 \%)$ \\
\hline \multicolumn{4}{|l|}{ Incision and drainage performed } \\
\hline No & 41 (71.9\%) & $16(28.1 \%)$ & $57(67.1 \%)$ \\
\hline Yes & $14(50.0 \%)$ & $14(50.0 \%)$ & $28(32.9 \%)$ \\
\hline \multicolumn{4}{|l|}{ Initial SSTI S aureus } \\
\hline No & $48(68.6 \%)$ & $22(31.4 \%)$ & $70(82.4 \%)$ \\
\hline Yes & $7(46.7 \%)$ & $8(26.7 \%)$ & $15(17.7 \%)$ \\
\hline \multicolumn{4}{|l|}{ Initial SSTI MRSA } \\
\hline No & $50(67.6 \%)$ & $24(32.4 \%)$ & $74(87.1 \%)$ \\
\hline Yes & $5(45.5 \%)$ & $6(54.5 \%)$ & $11(12.9 \%)$ \\
\hline \multicolumn{4}{|l|}{ Initial SSTI Treatment } \\
\hline Combination therapy & $11(55.0 \%)$ & $9(45.0 \%)$ & $20(23.5 \%)$ \\
\hline Beta-lactam & $12(60.0 \%)$ & $8(40.0 \%)$ & $20(23.5 \%)$ \\
\hline Clindamycin & 16 (76.2 \%) & $5(23.8 \%)$ & $21(24.7 \%)$ \\
\hline Fluoroquinolone & $4(80.0 \%)$ & $1(20.0 \%)$ & $5(5.9 \%)$ \\
\hline Trimethoprim- sulfamethoxazole & $5(62.5 \%)$ & $3(37.5 \%)$ & $8(9.4 \%)$ \\
\hline Vancomycin & $2(100 \%)$ & $0(0 \%)$ & $2(2.3 \%)$ \\
\hline None & $3(100 \%)$ & $0(0 \%)$ & $3(3.5 \%)$ \\
\hline Unknown & $2(33.3 \%)$ & $4(66.7 \%)$ & $6(7.1 \%)$ \\
\hline \multicolumn{4}{|l|}{ Chlamydia since 2004} \\
\hline No & $52(64.2 \%)$ & $29(35.8 \%)$ & $81(95.3 \%)$ \\
\hline Yes & $3(75.0 \%)$ & $1(25.0 \%)$ & $4(4.7 \%)$ \\
\hline \multicolumn{4}{|l|}{ Gonorrhea since 2004} \\
\hline No & $54(65.1 \%)$ & $29(34.9 \%)$ & $83(97.7 \%)$ \\
\hline Yes & $1(50.0 \%)$ & $1(50.0 \%)$ & $2(2.3 \%)$ \\
\hline \multicolumn{4}{|l|}{ Syphilis since 2004} \\
\hline No & $50(63.3 \%)$ & $29(36.7 \%)$ & 79 (92.9 \%) \\
\hline Yes & $5(83.3 \%)$ & 1 (16.7 \%) & $6(7.1 \%)$ \\
\hline \multicolumn{4}{|c|}{ Human papilloma virus (Clinical) since 2004} \\
\hline No & $47(65.3 \%)$ & $25(34.7 \%)$ & $72(84.7 \%)$ \\
\hline Yes & $8(61.5 \%)$ & $5(38.5 \%)$ & $13(15.3 \%)$ \\
\hline \multicolumn{4}{|c|}{ Human papilloma virus (Pap) since 2004} \\
\hline No & $45(64.3 \%)$ & $25(35.7 \%)$ & $70(82.4 \%)$ \\
\hline Yes & 10 (66.7\%) & 5 (33.3 \%) & 15 (27.6\%) \\
\hline
\end{tabular}


Table 1 Baseline characteristics of individuals who did and did not develop a recurrent SSTI during the study period (Continued)

\begin{tabular}{|c|c|c|c|}
\hline \multicolumn{4}{|c|}{ Trichomonas since 2004} \\
\hline No & $47(61.8 \%)$ & $29(38.2 \%)$ & $76(89.4 \%)$ \\
\hline Yes & $8(88.9 \%$ & $1(11.1 \%)$ & $9(10.6 \%)$ \\
\hline \multicolumn{4}{|c|}{ Any sexually transmitted disease since 2004} \\
\hline No & $29(59.2 \%)$ & $20(40.8 \%)$ & $49(57.7 \%)$ \\
\hline Yes & $26(72.2 \%)$ & $10(27.8 \%)$ & $36(42.4 \%)$ \\
\hline \multicolumn{4}{|c|}{ Pneumocystic jirovecii since 2004} \\
\hline No & $54(65.8 \%)$ & $28(34.2 \%)$ & $82(96.5 \%)$ \\
\hline Yes & $1(33.3 \%)$ & $2(66.7 \%)$ & $3(3.5 \%)$ \\
\hline \multicolumn{4}{|c|}{ Non-tuberculous mycobacteria since 2004} \\
\hline No & $53(63.9 \%)$ & 30 (36.1\%) & $83(97.7 \%)$ \\
\hline Yes & $2(100.0 \%)$ & $0(0.0 \%)$ & $2(2.4 \%)$ \\
\hline \multicolumn{4}{|c|}{ Cytomegalovirus since 2004} \\
\hline No & $54(64.3 \%)$ & $30(35.7 \%)$ & $84(98.8 \%)$ \\
\hline Yes & $1(100.0 \%)$ & $0(0.0 \%)$ & $1(1.2 \%)$ \\
\hline \multicolumn{4}{|c|}{ Cryptococcus since 2004} \\
\hline No & $53(64.6 \%)$ & 29 (35.4\%) & $82(96.5 \%)$ \\
\hline Yes & $2(66.7 \%)$ & $1(33.3 \%)$ & $3(3.5 \%)$ \\
\hline \multicolumn{4}{|c|}{ Initial TMP-SMX use } \\
\hline No & $41(65.1 \%)$ & 22 (34.9\%) & $63(74.1 \%)$ \\
\hline Yes & $14(63.6 \%)$ & $8(36.4 \%)$ & $22(25.9 \%)$ \\
\hline \multicolumn{4}{|c|}{ Initial azithromycin use } \\
\hline No & $48(63.2 \%)$ & $28(36.8 \%)$ & $76(89.4 \%)$ \\
\hline Yes & $7(77.8 \%)$ & $2(22.2 \%)$ & $9(10.6 \%)$ \\
\hline \multicolumn{4}{|c|}{ Initial HAART use } \\
\hline No & $13(52.0 \%)$ & $12(48.0 \%)$ & $25(29.4 \%)$ \\
\hline Yes & $42(70.0 \%)$ & 18 (30.0\%) & $60(70.6 \%)$ \\
\hline \multicolumn{4}{|c|}{ Initial catheter presence } \\
\hline No & $54(65.9 \%)$ & 28 (34.1\%) & $82(96.5 \%)$ \\
\hline Yes & $1(33.3 \%)$ & $2(66.7 \%)$ & $3(3.5 \%)$ \\
\hline \multicolumn{4}{|c|}{ Initial insurance Medicaid } \\
\hline No & $16(64.0 \%)$ & $9(36.0 \%)$ & $25(29.4 \%)$ \\
\hline Yes & $39(65.0 \%)$ & $21(35.0 \%)$ & $60(70.6 \%)$ \\
\hline \multicolumn{4}{|c|}{ Initial cancer status } \\
\hline No & $50(63.3 \%)$ & 29 (36.7 \%) & 79 (92.9 \%) \\
\hline Yes & $5(83.3 \%)$ & $1(16.7 \%)$ & $6(7.1 \%)$ \\
\hline \multicolumn{4}{|c|}{ Initial diabetes } \\
\hline No & $48(65.8 \%)$ & 25 (34.3\%) & $73(85.9 \%)$ \\
\hline Yes & $7(58.3 \%)$ & $5(41.7 \%)$ & $12(14.1 \%)$ \\
\hline \multicolumn{4}{|c|}{ Initial ESRD status } \\
\hline No & $48(65.8 \%)$ & 25 (34.3\%) & $73(85.9 \%)$ \\
\hline Yes & 7 (58.3 \%) & 5 (41.7 \%) & 12 (14.1 \%) \\
\hline
\end{tabular}




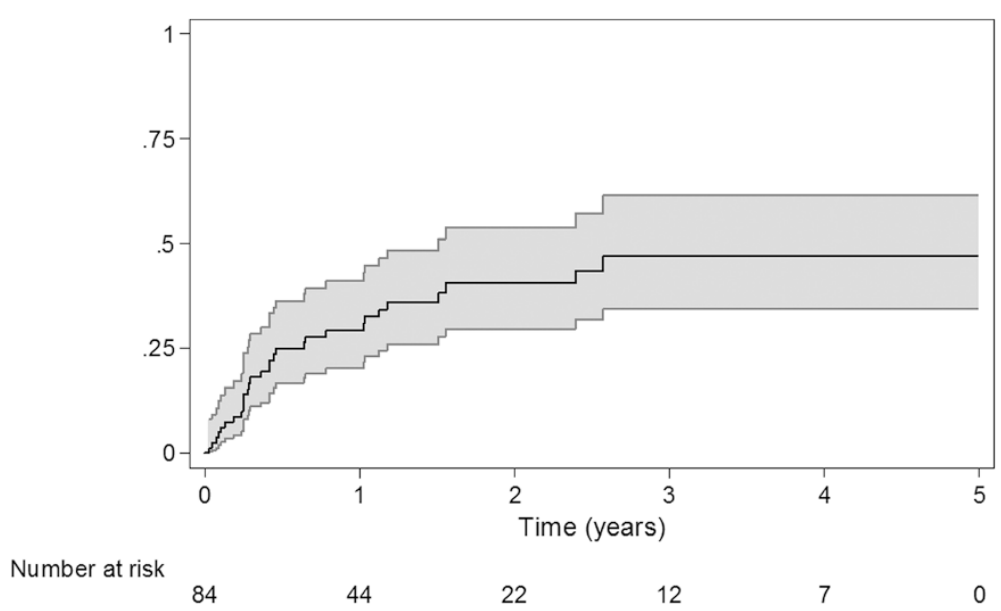

Fig. 1 Kaplan-Meier curve demonstrating the cumulative risk of recurrent SSTI over time with $95 \%$ confidence intervals

liver disease (HR 2.89; $95 \%$ CI 1.00-8.35, $p=0.05$ ), and lymphedema (HR 4.12; $95 \%$ CI 1.24-14.1; $p=0.02$ ) were associated with an increased risk of recurrent SSTI, while African-American race was associated with decreased risk of recurrent SSTI (HR 0.34; $95 \%$ CI 0.11-1.00; $p=0.05$ ).

Predictors with $p<0.2$ were candidates for the final multivariate Cox model, which was developed by a forward selection algorithm. Significant predictors as well as theoretical concerns led to the choice of our final model for multivariate regression. For example, we found a strong association between lymphedema and intravenous drug use (IVDU) in our cohort; we accordingly only included IVDU in the final model to avoid collinearity.

Table 2 Proportion of patient-days with exposure to timevarying covariates

\begin{tabular}{ll}
\hline Time-varying exposure & $\begin{array}{l}\text { Percentage of days of } \\
\text { follow-up with exposure }\end{array}$ \\
\hline Trimethoprim-sulfamethoxazole & $24.7 \%$ \\
Azithromycin & $12.0 \%$ \\
HAART & $77.1 \%$ \\
Catheter & $1.6 \%$ \\
Public Aid & $79.8 \%$ \\
Viral load & \\
$<1000$ copies $/ \mathrm{mL}$ & $60.0 \%$ \\
$1000+$ copes $/ \mathrm{mL}$ & $40.0 \%$ \\
CD4 & \\
$<50$ cells $/ \mathrm{mL}$ & $6.5 \%$ \\
$50-99$ cells $/ \mathrm{mL}$ & $4.0 \%$ \\
$100+$ cells $/ \mathrm{mL}$ & $89.5 \%$ \\
Cancer & $7.8 \%$ \\
Diabetes & $14.6 \%$ \\
End-stage renal disease & $11.5 \%$ \\
\hline
\end{tabular}

Similarly, although gonorrhea was a strong statistical predictor of recurrent SSTI in several preliminary multivariate models, we found that models including gonorrhea were overly influenced by the single subject with a history of gonorrhea during follow up with recurrent SSTI (out of two subjects with a history of gonorrhea during follow up). Accordingly, gonorrhea was not included in the final model as it was felt that the data were inadequate to allow for a multivariable analysis including this predictor. Risk factors for recurrent SSTI in the final multivariable Cox regression model were non-hepatitis liver disease (HR 3.44; $95 \%$ CI 1.02-11.5; $p=0.05)$, the presence of an intravenous catheter (HR 6.50; $95 \%$ CI 1.47-28.7; $p=0.01$ ), and a history of intravenous drug use (HR 2.80; $95 \%$ CI 1.02-7.65; $p=0.05)$; African-American race remained associated with a decreased risk (HR 0.17; $95 \%$ CI 0.05-0.54; $p<0.01$ ). A trend was present for HIV viral load $\geq 1000$ copies $/ \mathrm{mL}$ as an independent risk factor for recurrent SSTI (HR 2.21; $95 \%$ CI 0.99-4.94; $p=0.05$ ), which was nearly statistically significant. A Cox-Snell residual plot demonstrated that the model fit the data well (plot not shown).

As a sensitivity analysis, we repeated the above analyses but used 30-, 60-, 90- and 365-day recent averages for a number of the above time-varying factors, without significant change in the results (data not shown). Similarly, we used linearly extrapolated CD4+ counts and HIV VLs and found that point estimates did not change significantly. As a final sensitivity analysis, we used CD4 + count of 200 as an alternative cutoff, without significant change in the results.

\section{Discussion}

In an urban, primarily African-American cohort, we confirmed that patients infected with HIV who had a medically attended SSTI had a high rate of subsequent SSTI, with most recurrences diagnosed within months 
Table 3 Predictors of risk of recurrent SSTI in univariate and multivariate Cox regression models

\begin{tabular}{|c|c|c|c|c|}
\hline \multirow[t]{2}{*}{ Factor } & Univariate HR & $p$-value & $\begin{array}{l}\text { Multivariate HR } \\
\text { (Final model) }\end{array}$ & $p$-value \\
\hline & \multicolumn{2}{|l|}{$(95 \% \mathrm{Cl})$} & \multicolumn{2}{|l|}{$(95 \%$ Cl) } \\
\hline HAART & $0.67(0.31-1.44)$ & 0.30 & & \\
\hline $\begin{array}{l}\text { Trimethoprim- } \\
\text { sulfamethoxazole }\end{array}$ & $0.73(0.28-1.91)$ & 0.52 & & \\
\hline Azithromycin & $1.38(0.48-3.98)$ & 0.55 & & \\
\hline Age (cont) & $0.99(0.96-1.03)$ & 0.71 & & \\
\hline STD (any) & $1.06(0.49-2.27)$ & 0.88 & & \\
\hline Chlamydia & $1.18(0.16-8.77)$ & 0.87 & & \\
\hline Gonorrhea & $4.96(0.65-37.7)$ & 0.12 & & \\
\hline Syphilis & $0.49(0.07-3.59)$ & 0.48 & & \\
\hline $\begin{array}{l}\text { Human } \\
\text { papilloma } \\
\text { virus (clinical) }\end{array}$ & $1.58(0.60-4.17)$ & 0.35 & & \\
\hline $\begin{array}{l}\text { Human } \\
\text { papilloma } \\
\text { virus (Pap) }\end{array}$ & $1.12(0.43-2.92)$ & 0.82 & & \\
\hline Trichomonas & $0.45(0.06-3.33)$ & 0.44 & & \\
\hline Catheter & $4.62(1.08-19.7)$ & 0.04 & $6.50(1.47-28.7)$ & 0.01 \\
\hline Medicaid & $0.64(0.30-1.37)$ & 0.25 & & \\
\hline $\begin{array}{l}\text { End stage } \\
\text { renal disease }\end{array}$ & $0.70(0.16-2.93)$ & 0.62 & & \\
\hline Cancer & $0.51(0.07-3.73)$ & 0.51 & & \\
\hline Diabetes & $1.03(0.39-2.69)$ & 0.96 & & \\
\hline Hepatitis B & $1.27(0.38-4.20)$ & 0.69 & & \\
\hline Hepatitis C & $\begin{array}{l}0.91 \\
(0.32-2.61)\end{array}$ & 0.86 & & \\
\hline Incarceration & $0.22(0.03-1.65)$ & 0.14 & & \\
\hline $\begin{array}{l}\text { Other chronic } \\
\text { liver disease }\end{array}$ & $2.89(1.00-8.35)$ & 0.05 & $3.43(1.02-11.5)$ & 0.05 \\
\hline $\begin{array}{l}\text { Men who } \\
\text { have sex } \\
\text { with men }\end{array}$ & $0.81(0.36-1.81)$ & 0.60 & & \\
\hline IVDU & $2.41(0.98-5.91)$ & 0.06 & $2.80(1.02-7.65)$ & 0.05 \\
\hline Male & $0.87(0.42-1.79)$ & 0.71 & & \\
\hline $\begin{array}{l}\text { African-American } \\
\text { race }\end{array}$ & $\begin{array}{l}0.34 \\
(0.11-1.00)\end{array}$ & 0.05 & $0.12(0.03-0.41)$ & $<0.01$ \\
\hline $\begin{array}{l}\text { Chronic skin } \\
\text { disease: Any }\end{array}$ & $\begin{array}{l}1.36 \\
(0.66-2.79)\end{array}$ & 0.41 & & \\
\hline Psoriasis & $\begin{array}{l}0.46 \\
(0.06-3.39)\end{array}$ & 0.45 & & \\
\hline Lymphedema & $\begin{array}{l}4.12 \\
(1.24-14.1)\end{array}$ & 0.02 & & \\
\hline \multicolumn{5}{|l|}{ CD4 } \\
\hline $100+$ & Reference & & & \\
\hline$<100$ & $\begin{array}{l}1.13 \\
(0.39-3.24)\end{array}$ & 0.83 & & \\
\hline
\end{tabular}

Table 3 Predictors of risk of recurrent SSTI in univariate and multivariate Cox regression models (Continued)

\begin{tabular}{|c|c|c|c|c|}
\hline \multicolumn{5}{|l|}{ Viral load } \\
\hline$<1000$ & Reference & & Reference & \\
\hline $1000+$ & 1.58 & 0.22 & $2.21(0.99-4.94)$ & 0.05 \\
\hline \multicolumn{5}{|l|}{ Initial SSTI type } \\
\hline Abscess & Reference & & & \\
\hline Furuncle & $0.26(0.03-1.91)$ & 0.19 & & \\
\hline Cellulitis & $\begin{array}{l}0.66 \\
(0.28-1.55)\end{array}$ & 0.34 & & \\
\hline $\begin{array}{l}\text { Incision and } \\
\text { drainage performed } \\
\text { on initial SSTI }\end{array}$ & $1.69(0.82-3.47)$ & 0.15 & & \\
\hline $\begin{array}{l}\text { Initial SSTI } \\
\text { S. aureus }\end{array}$ & $2.10(0.93-4.72)$ & 0.07 & & \\
\hline $\begin{array}{l}\text { Initial SSTI } \\
\text { MRSA }\end{array}$ & $2.21(0.90-5.40)$ & 0.08 & & \\
\hline
\end{tabular}

of the initial SSTI. We identified risk factors for recurrence which do not entirely overlap the risk factors for initial SSTI found in our previous paper [19] or in the work of others [20], though our studies differed from most other studies in examining all subjects with SSTIs, not just those with proven MRSA SSTIs. It may be that the risk factors for initial SSTI occurrence differ from those that predispose to continued colonization with or re-exposure to pathogenic bacteria.

In our cohort, African Americans, who constituted more than $90 \%$ of the patient sample, demonstrated a significantly lower rate of recurrent SSTI as compared with others. We surmise that the risk factors leading to initial SSTIs in those not of African-American descent in our cohort may be more strongly associated with the risk of SSTI recurrence than the risk factors leading to initial SSTI in African Americans. However, our sample of non-African Americans is too small to permit rigorous testing of this hypothesis. Previous observational cohorts have noted that African-Americans are less likely to remain in care [21], but differential rates of follow-up for African-Americans would only explain our results if African Americans having recurrent SSTIs were more likely than other African Americans to be lost to followup. We also found that established risk factors for SSTI recurrence such as intravenous drug use and presence of an intravenous catheter [8] predicted SSTI recurrence.

Some of our preliminary modelling suggested an independent impact of a gonorrhea diagnosis, although the STD incidence observed in our population was too low to assess reliably the effect of STD diagnosis on SSTI recurrence risk. It has been proposed that ongoing high risk sexual behavior may re-expose HIV-infected patients to pathogenic strains of MRSA. Prior studies by other researchers have found that many HIV-infected 
patients only demonstrate groin colonization with MRSA without colonization at other sites [22]. The occurrence of gonorrhea or other STDs may serve as a marker of continuing exposure to other colonized individuals, leading to reacquisition of pathogenic strains and predisposing towards SSTI recurrence.

We found that non-hepatitis liver disease, which in our cohort was predominantly due to alcoholic liver disease, was a risk factor for recurrent SSTI. Although not previously described specifically as a risk factor for recurrent SSTI in HIV-infected populations, the association between cirrhosis and SSTI risk has been previously described by other authors [23].

We were unable to test the finding of others that treatment of the initial SSTI with minocycline reduces the risk of recurrence [11] because only one patient in our study received minocycline as the treatment for the initial SSTI, which reflects institutional preferences for certain antimicrobials for the treatment of SSTIs at our center.

Our finding that lack of virologic control, better than CD4+ lymphocyte count per se, predicted SSTI risk is difficult to explain but is consistent with the findings of other authors [10]. This effect may be related to the immunologic effect of uncontrolled HIV replication. Alternatively, unsuppressed viral load, a marker of poor adherence to medication, may serve as a marker of patients who are not taking trimethoprim-sulfamethoxazole prophylaxis to prevent pneumocystis pneumonia, which potentially may also be protective against SSTI [24, 25]. Alternatively, lack of virologic suppression may be a marker of other behavioral and demographic risk factors for SSTI.

Our study is one of only six in which authors have examined SSTI recurrence in HIV-infected adults (Table 4). Skiest et al. performed a single-center prospective study at an urban, public clinic in Dallas, accruing patients with initial S. aureus skin infection seeking outpatient care in 2003-2004 [12]. Recurrent SSTI was noted in 11/37 patients $(29.7 \%)$. Adjustment for variable length of followup after the initial SSTI was not performed. Whether or not appropriate antimicrobial therapy was prescribed and whether or not incision and drainage was performed did not affect recurrence risk. No additional statistical analysis of risk factors for recurrence was reported.

Graber et al. performed a single-center retrospective study at an urban, public clinic in San Francisco, accruing patients with initial MRSA infection seeking outpatient care in 2002-2006 [9]. Patients were followed through 2007. A recurrent SSTI occurred in $44 / 62$ patients $(71.0 \%)$. Adjustment for variable length of followup after the initial SSTI was not performed. The median time to recurrence was 135.5 days, which was similar to the finding in our study. Cox regression performed on demographic and clinical predictors did not yield a significant predictor of SSTI recurrence. Of 62 patients with an initial MRSA SSTI, 17 (27 \%) developed a recurrence.

Vyas et al. performed a single-center retrospective study at a military-affiliated HIV clinic in San Diego, accruing patients with initial MRSA SSTI in 2003-2010 $[7,11]$. Patients were followed through 2010. Of 63 patients with initial MRSA SSTI, 17 (27 \%) experienced a recurrent MRSA SSTI. Adjustment for variable length of follow-up after the initial SSTI was not performed. The lower rate of recurrence in this study may have resulted from the inclusion of only culture-proven recurrences. Incision and drainage was not associated with a decreased risk of recurrence. Use of an antibiotic other than minocycline and hospitalization for the initial SSTI were significant risk factors for recurrence in a multivariable analysis (Table 4). Patients with a recurrent SSTI demonstrated a significantly lower CD4+ count in a mixed-effects model using CD4+ lymphocyte count as the outcome.

Crum-Cianflone et al. performed a single-center retrospective study at a military-affiliated HIV clinic in San Diego, accruing patients with initial MRSA SSTI in 2000-2007 [10]. This study was performed at the same center as Vyas's study in an overlapping time frame. Of 31 patients with initial MRSA SSTI, 14 (41 \%) had a recurrent SSTI. The proportion of recurrence in this study differed from that observed in the Vyas study as the outcome included all SSTIs, not just SSTIs proven to be caused by MRSA. Median time to recurrence was 4 months, comparable to the results of our study. In a multivariable logistic regression model, only HIV VL > 1000 copies/mL was a significant predictor of recurrence.

Crum-Cianflone et al. examined data from the US Military HIV Natural History Study, a multicenter prospective cohort study, from 2006 to 2010 [26]. Of 379 subjects with an initial SSTI, $31 \%$ experienced a recurrence at a median 7 months after the initial SSTI. They did not identify a statistically significant risk factor for recurrent SSTI. However, there was some suggestion in the data that the presence of a dermatological condition at the time of diagnosis of the initial SSTI (HR 1.99; $95 \%$ CI 0.95-4.16; $p=0.07$ ) and higher HIV RNA level (HR 1.18 per 10-fold increase in viral load; $95 \%$ CI 0.98-1.43; $p=$ $0.08)$ may have been associated with recurrence.

Our study was limited as it was a single-center retrospective medical record review. Therefore, some demographic information was missing. The diagnosis and outcome of SSTI was based on physician documentation. Older paper records were incomplete. SSTIs diagnosed at centers other than our own were missed, and so our estimate of SSTI recurrence risk was likely a lower bound. We have no specific data on how often patients in our cohort sought care elsewhere. There are community hospitals with emergency departments but no other major 
Table 4 Summary of studies examining the incidence of and risk factors for recurrent SSTI in HIV-infected adults

\begin{tabular}{|c|c|c|c|c|c|}
\hline Study & Population & Methodology & Outcome & $\begin{array}{l}\text { Recurrence } \\
\text { rate }\end{array}$ & $\begin{array}{l}\text { Risk factors } \\
\text { for recurrence }\end{array}$ \\
\hline \multirow[t]{4}{*}{ Crum-Cianflone et al. [10] } & \multirow[t]{4}{*}{$\begin{array}{l}31 \text { subjects with } \\
\text { initial MRSA SSTI }\end{array}$} & \multirow[t]{4}{*}{$\begin{array}{l}\text { Retrospective, } \\
\text { single center }\end{array}$} & \multirow[t]{4}{*}{ Any recurrent SSTI } & $\begin{array}{l}41 \% \\
\text { (all SSTI) }\end{array}$ & Lower CD4 count ${ }^{a}$ \\
\hline & & & & \multirow{3}{*}{$\begin{array}{l}21 \% \\
\text { (MRSA SSTI) }\end{array}$} & Higher viral load ${ }^{a b}$ \\
\hline & & & & & Lack of incision and drainage ${ }^{a b}$ \\
\hline & & & & & Positive nare culture ${ }^{a}$ \\
\hline \multirow[t]{2}{*}{ Crum-Cianflone et al. [26] } & \multirow[t]{2}{*}{$\begin{array}{l}379 \text { subjects with } \\
\text { initial SSTI }\end{array}$} & \multirow[t]{2}{*}{$\begin{array}{l}\text { Prospective, } \\
\text { multi-center }\end{array}$} & \multirow[t]{2}{*}{ Any recurrent SSTI } & \multirow[t]{2}{*}{$31 \%$} & $\begin{array}{l}\text { No significant risk factor } \\
\text { identified }\end{array}$ \\
\hline & & & & & $\begin{array}{l}\text { Trend toward viral load and } \\
\text { dermatologic condition } \\
\text { predicting SSTI recurrence }\end{array}$ \\
\hline Graber et al. [9] & $\begin{array}{l}62 \text { subjects with } \\
\text { initial MRSA SSTI }\end{array}$ & $\begin{array}{l}\text { Retrospective, } \\
\text { single center }\end{array}$ & Any recurrent SSTI & $71 \%$ & $\begin{array}{l}\text { No significant risk factor } \\
\text { identified }\end{array}$ \\
\hline \multirow[t]{10}{*}{ Vyas et al. [11] } & \multirow{10}{*}{$\begin{array}{l}63 \text { individuals with } \\
\text { initial MRSA SSTI }\end{array}$} & \multirow{10}{*}{$\begin{array}{l}\text { Retrospective, } \\
\text { single center }\end{array}$} & \multirow[t]{10}{*}{ Recurrent MRSA SSTI } & \multirow[t]{10}{*}{$27 \%$} & Older age ${ }^{a}$ \\
\hline & & & & & $\begin{array}{l}\text { Lower CD4 count at } \\
\text { initial SSTI }\end{array}$ \\
\hline & & & & & Lower nadir CD4 count $^{\mathrm{a}}$ \\
\hline & & & & & Higher peak viral load ${ }^{a}$ \\
\hline & & & & & Prior AIDS defining illness ${ }^{a}$ \\
\hline & & & & & History of malignancy ${ }^{a}$ \\
\hline & & & & & $\mathrm{IVDU}^{*}$ \\
\hline & & & & & Use of antibiotic other than minocycline $e^{\mathrm{ab}}$ \\
\hline & & & & & Hospital admission ${ }^{\mathrm{ab}}$ \\
\hline & & & & & Surgery ${ }^{a}$ \\
\hline Skiest et al. [12] & $\begin{array}{l}41 \text { subjects with initial } \\
\text { MRSA SSTI }\end{array}$ & $\begin{array}{l}\text { Prospective, } \\
\text { single center }\end{array}$ & Any recurrent SSTI & $29.7 \%$ & $\begin{array}{l}\text { No significant risk factor } \\
\text { identified }\end{array}$ \\
\hline \multirow[t]{5}{*}{ Present study } & \multirow{5}{*}{$\begin{array}{l}85 \text { subjects with } \\
\text { initial SSTI }\end{array}$} & \multirow{5}{*}{$\begin{array}{l}\text { Retrospective, } \\
\text { single center }\end{array}$} & \multirow[t]{5}{*}{ Any recurrent SSTI } & \multirow[t]{5}{*}{$35 \%$ (raw) } & Catheter $^{\mathrm{ab}}$ \\
\hline & & & & & Non-viral liver disease $^{a b}$ \\
\hline & & & & & Non-African American race ${ }^{\mathrm{ab}}$ \\
\hline & & & & & Lymphedema $^{a}$ \\
\hline & & & & & IVDU ${ }^{b}$ \\
\hline
\end{tabular}

tertiary care centers within five miles of our center. Cox regression requires an assumption of non-informative censoring (i.e., that subjects who are lost to follow-up are similar in their characteristics to those who remain engaged in care), and the results of our analysis may be biased to the extent that this assumption is violated. However, the problem of loss to follow-up is inherent to any study of urban HIV-infected populations [27].

\section{Conclusions}

This study contributes to the literature as one of the few analyses of risk factors for recurrence of SSTI in an urban, socioeconomically diverse cohort of HIV-infected adults. Our study is unique in that most previous studies on this topic have only included subjects whose initial SSTIs were culture-proven S. aureus SSTIs, while our study includes all subjects in our cohort with an initial SSTI. Our investigation improves on other studies by explicitly accounting for time-varying predictor variables and for variable time of follow-up using Kaplan-Meier methods and by following patients for a relatively long period of time. Further work to define risk factors for SSTI recurrence will enable physicians to target potential preventative interventions to those HIV-infected individuals at highest risk of SSTI recurrence.

\section{Abbreviations}

MRSA: Methicillin-resistant Staphylococcus aureus; CA-MRSA: Communityassociated methicillin-resistant Staphylococcus aureus; SSTI: Skin and soft tissue infection; STD: Sexually transmitted disease; HIV: Human immunodeficiency virus; VL: Viral load; HR: Hazard ratio; Cl: Confidence interval; IVDU: Intravenous drug use; HAART: Highly active antiretroviral therapy; OI: Opportunistic infection; IQR: Interquartile range; CA-

SSTI: Community-associated skin and soft tissue infection. 


\section{Competing interests}

The authors declare that they have no competing interests.

\section{Authors' contributions}

MZD conceived of the study. $\mathrm{VH}$ and MZD designed the study. $\mathrm{VH}, \mathrm{MM}$, and ES collected the data. $\mathrm{VH}$ and MZD performed the statistical analysis. All authors contributed to data analysis and interpretation. VH wrote the first manuscript draft. All authors contributed to the final manuscript. All authors read and approved the final manuscript.

\section{Acknowledgements}

Richard Rodgers provided invaluable assistance in the data collection process. Preliminary data from this study were presented as an abstract at ID Week 2014, Philadelphia, PA, October 8-12, 2014.

Michael David acknowledges funding from NIH NIAID grant K23 Al095361

\section{Author details}

${ }^{1}$ Division of Infectious Diseases, Department of Medicine, Baylor College of Medicine, 6620 Main, Suite 1375, Houston, TX 77030, USA. ²Department of Medicine, University of Chicago, 5841 S Maryland, Chicago, IL 60637, USA. ${ }^{3}$ Department of Pediatrics and Department of Public Health Sciences, University of Chicago, 5841 S. Maryland Ave. MC6054, Chicago 60637IL, USA.

Received: 17 March 2015 Accepted: 14 October 2015

Published online: 26 October 2015

\section{References}

1. Herold BC, Immergluck LC, Maranan MC, Lauderdale DS, Gaskin RE, Boyle-Vavra S, et al. Community-acquired methicillin-resistant Staphylococcus aureus in children with no identified predisposing risk. JAMA. 1998;279(8):593-8.

2. King MD, Humphrey BJ, Wang YF, Kourbatova EV, Ray SM, Blumberg HM. Emergence of community-acquired methicillin-resistant Staphylococcus aureus USA 300 clone as the predominant cause of skin and soft-tissue infections. Ann Intern Med. 2006;144(5):309-17.

3. David MZ, Daum RS. Community-associated methicillin-resistant Staphylococcus aureus: epidemiology and clinical consequences of an emerging epidemic. Clin Microbiol Rev. 2010;23(3):616-87. doi:10.1128/ cmr.00081-09.

4. Imaz A, Pujol M, Barragan P, Dominguez MA, Tiraboschi JM, Podzamczer D. Community associated methicillin-resistant Staphylococcus aureus in HIV-infected patients. AIDS Rev. 2010;12(3):153-63.

5. Cole J, Popovich K. Impact of community-associated methicillin resistant Staphylococcus aureus on HIV-infected patients. Curr HIV/AIDS Rep. 2013;10(3):244-53. doi:10.1007/s11904-013-0161-0.

6. Hemmige V, McNulty M, Silverman E, David MZ. Predictors of skin and soft tissue infections in HIV-infected outpatients in the community-associated methicillin-resistant Staphylococcus aureus era. Eur J Clin Microbiol Infect Dis. 2015;34(2):339-47. doi:10.1007/s10096-014-2237-1.

7. Vyas K, Hospenthal DR, Mende K, Crum-Cianflone NF. Recurrent communityacquired methicillin-resistant Staphylococcus aureus infections in an HIV-infected person. J Clin Microbiol. 2011;49(5):2047-53. doi:10.1128/ jcm.02423-10.

8. Anderson EJ, Hawkins C, Bolon MK, Palella Jr FJ. A series of skin and soft tissue infections due to methicillin-resistant Staphylococcus aureus in HIV-infected patients. J Acquir Immune Defic Syndr. 2006;41(1):125-7.

9. Graber CJ, Jacobson MA, Perdreau-Remington F, Chambers HF, Diep BA. Recurrence of skin and soft tissue infection caused by methicillin-resistant Staphylococcus aureus in a HIV primary care clinic. J Acquir Immune Defic Syndr. 2008;49(2):231-3. doi:10.1097/QAl.0b013e318183a947.

10. Crum-Cianflone N, Weekes J, Bavaro M. Recurrent community-associated methicillin-resistant Staphylococcus aureus infections among HIV-infected persons: incidence and risk factors. AIDS Patient Care STDS. 2009:23(7):499-502. doi:10.1089/apc.2008.0240.

11. Vyas KJ, Shadyab AH, Lin CD, Crum-Cianflone NF. Trends and Factors Associated with Initial and Recurrent Methicillin-Resistant Staphylococcus aureus (MRSA) Skin and Soft-Tissue Infections among HIV-Infected Persons: An 18-Year Study. Journal of the International Association of Providers of AIDS Care. 2013. doi:10.1177/2325957412473780.
12. Skiest D, Brown K, Hester J, Moore T, Crosby C, Mussa HR, et al. Communityonset methicillin-resistant Staphylococcus aureus in an urban HIV clinic. HIV Med. 2006;7(6):361-8. doi:10.1111/j.1468-1293.2006.00394.x.

13. Cox DR. Regression models and life tables. JR Stat Soc B. 1972;34(2):187-220.

14. Cox DR, Snell EJ. A general definition of residuals (with discussion). J R Stat Soc Ser B. 1968;30:248-75.

15. Cleves M, Gould W, Gutierrez R, Marchenko Y. An Introduction to Survival Analysis Using Stata. 3rd ed. College Station, TX: Stata Press; 2010.

16. Yelland LN, Salter AB, Ryan P. Performance of the modified poisson regression approach for estimating relative risks from clustered prospective data. Am J Epidemiol. 2011;174(8):984-92. doi:10.1093/aje/kwr183.

17. Hosmer D, Lemeshow S. Applied survival analysis: regression modeling of time to event data. Hoboken, NJ: Wiley; 2011.

18. Kallen AJ, Mu Y, Bulens S, Reingold A, Petit S, Gershman K, et al. Health care-associated invasive MRSA infections, 2005-2008. JAMA. 2010;304(6):641-8. doi:10.1001/jama.2010.1115.

19. Hemmige V, McNulty M, Silverman E, David MZ. Predictors of skin and soft tissue infections in HIV-infected outpatients in the community-associated methicillin-resistant Staphylococcus aureus era. Eur J Clin Microbiol Infect Dis. 2014. doi:10.1007/s10096-014-2237-1.

20. Hidron Al, Kempker R, Moanna A, Rimland D. Methicillin-resistant Staphylococcus aureus in HIV-infected patients. Infection and Drug Resistance. 2010;3:73-86.

21. Mugavero MJ, Lin HY, Willig JH, Westfall AO, Ulett KB, Routman JS, et al. Missed visits and mortality among patients establishing initial outpatient HIV treatment. Clin Infect Dis. 2009;48(2):248-56. doi:10.1086/595705.

22. Popovich K, Hota B, Aroutcheva A, Kurien L, Patel J, Lyles-Banks R, et al. Community-Associated Methicillin-Resistant Staphylococcus aureus Colonization Burden in HIV-Infected Patients. Clin Infect Dis. 2013;56(8):1067-74. doi:10.1093/cid/cit010.

23. Corredoira JM, Ariza J, Pallarés R, Carratalá J, Viladrich PF, Rufí G, et al. Gramnegative bacillary cellulitis in patients with hepatic cirrhosis. Eur J Clin Microbiol Infect Dis. 1994;13(1):19-24. doi:10.1007/BF02026118.

24. Mathews WC, Caperna JC, Barber RE, Torriani FJ, Miller LG, May S, et al. Incidence of and risk factors for clinically significant methicillin-resistant Staphylococcus aureus infection in a cohort of HIV-infected adults. J Acquir Immune Defic Syndr. 2005;40(2):155-60.

25. Lee NE, Taylor MM, Bancroft E, Ruane PJ, Morgan M, McCoy L, et al. Risk factors for community-associated methicillin-resistant Staphylococcus aureus skin infections among HIV-positive men who have sex with men. Clin Infect Dis. 2005;40(10):1529-34. doi:10.1086/429827.

26. Crum-Cianflone NF, Grandits G, Weintrob A, Ganesan A, Agan B, Landrum M. Skin and soft tissue infections among HIV-infected persons in the late combination antiretroviral therapy era. Int J STD AIDS. 2012;23(7):507-11. doi:10.1258/ijsa.2011.011360.

27. Giordano TP, Gifford AL, White Jr AC, Suarez-Almazor ME, Rabeneck L, Hartman C, et al. Retention in care: a challenge to survival with HIV infection. Clin Infect Dis. 2007:44(11):1493-9. doi:10.1086/516778.

\section{Submit your next manuscript to BioMed Central and take full advantage of:}

- Convenient online submission

- Thorough peer review

- No space constraints or color figure charges

- Immediate publication on acceptance

- Inclusion in PubMed, CAS, Scopus and Google Scholar

- Research which is freely available for redistribution 